\title{
A case of paraplegia due to limb girdle muscular dystrophy with coexisting gitelman's syndrome
}

\author{
Robin George Manappallil \\ Consultant - Physician, Department of Medicine, National Hospital, Calicut, Kerala 673009, India
}

A B S T R A C T

\begin{abstract}
Muscular dystrophies are a rare group of disorders affecting the skeletal muscles, which are progressive, hereditary and degenerative. A sudden worsening of the condition should raise the possibility of hypokalemia, followed by its evaluation and correction. The patient being reported is a 33 year old lady who presented with generalized but mainly bilateral lower limb proximal muscle weakness; and was diagnosed to have limb girdle muscular dystrophy (LGMD), with the support of muscle biopsy and electromyography (EMG) study. However, her laboratory investigations showed hypokalemia, hypomagnesemia and hypocalciuria, suggestive of an underlying Gitelman's syndrome (GS). Such a scenario has rarely been reported.
\end{abstract}

Key words: Limb girdle muscular dystrophy, Gitelman's syndrome, Proximal muscle weakness, Hypokalemia, Hypomagnesimia

\section{INTRODUCTION}

Limb Girdle Muscular Dystrophy (LGMD) is a syndrome which represents more than one disorder, the only unifying feature being the presence of limb-girdle weakness with sparing of the facial muscles. The inheritance pattern is variable, but autosomal recessive forms are more common. The onset ranges from late in the first decade to fourth decade, affecting both males and females. Gitelman's syndrome (GS) is an autosomal recessive disorder of renal tubules, characterized by hypokalemic metabolic alkalosis, hypomagnesemia and hypocalciuria. They present during adolescence or adulthood, with complaints of proximal muscle weakness, but are usually episodic in nature and triggered by meals high in carbohydrate or sodium. The patient being reported here is a 33 year old lady who presented with generalized but mainly bilateral lower limb proximal muscle weakness and was diagnosed to have LGMD. However, her laboratory investigations were suggestive of a coexisting GS. Following treatment of GS, there was improvement in her muscle weakness.

\section{CASE HISTORY}

This is a case of a 33 year old female, a homemaker from a rural area in Kerala, India, who presented with history of bilateral lower limb weakness for the last 3 years and generalized weakness since 10 days. Her lower limb weakness had also increased over these 10 days. She did not complain of upper limb weakness and was able to do her routine work with her shoulder, arms and hands. She used to walk in house with the support of the walls. She did not give history of slipping of footwear or any sensory deficits. The disease was progressive in nature initially, but has remained static over the past 1 year. However, over the past 10 days she has been experiencing increased weakness of her lower limbs and generalized fatigue. This was not associated with any muscle tenderness or spasms. There was no cranial nerve or sensory involvement. There was no history of any trauma or fever or other systemic involvement.

She did not have any co-morbidity like diabetes, hypertension or thyroid disorders and was not on any medications like 
steroids or statins. She has a child who is 4 years old. Her symptoms started following abortion of her second fetus 3 years ago. There was no history of spinal anaesthesia. The patient was born of non-consanguineous marriage, with normal developmental milestones. Her father also had history of lower limb weakness and died at the age of 40 (details unknown).

On examination, she was moderately built, conscious, oriented and afebrile. Her heart rate was 76 beats/minute, blood pressure of $90 / 60 \mathrm{mmHg}$ and respiratory rate $20 /$ minute. She did not have any pallor, icterus, cyanosis, clubbing or lymphadenopathy. Neurological examination revealed atrophy of bilateral thigh muscles with pseudo hypertrophy of her calf muscles. Power was $2 / 5$ at both hip joints, $3 / 5$ at both knee joints and $5 / 5$ at both ankles. Her lower limb deep tendon reflexes were diminished. Fasciculations were absent. Plantars were bilaterally flexor. Gower's sign was positive. Her upper limb power and reflexes were preserved. All superficial reflexes were present. There was no cranial nerve, sensory or cerebellar involvement. Skull and spine were normal. Other systemic examinations were also normal.

Her blood investigations showed normal complete blood count, renal and liver functions. Her thyroid profile, lipid profile, sugars and $\mathrm{HbA1c}$ were normal. She had severe hypokalemia of $1.8 \mathrm{mEq} / \mathrm{L}(3.5-5)$. Her creatinine phosphokinase levels was within normal limit, 26 IU/L $(24-190)$. ECG, ECHO heart, USG abdomen and chest Xray were normal. ANA and Anti dsDNA were negative. Nerve conduction studies were normal. CT brain, MRI spine and CSF analysis were normal. EMG of right lower limb showed myopathic pattern. Muscle biopsy from right quadriceps showed degeneration of fibres with necrosis and inflammatory infiltrates. Genetic studies and DNA analysis could not be done (due to lack of facilities and financial constraints). Based on her history, clinical examination and investigation reports, the diagnosis of LGMD with hypokalemia was considered.

She was started on oral potassium correction. However, her potassium levels continued to be very low. Her urine potassium was $50 \mathrm{mmol} /$ day, suggestive of renal loss. Urine microscopy, specific gravity, osmolality and urine sodium were normal. ABG showed metabolic alkalosis and urine chloride levels were $415 \mathrm{mmol} /$ day. There was no history of thiazide use. Serum cortisol and aldosterone levels were normal. She had hypomagnesemia of $1 \mathrm{mg} \%(1.5-2.3)$, hypocalcemia of $7.8 \mathrm{mg} \%(8.7-10.2)$ and hypocalciuria $96 \mathrm{mg} /$ day (100-300). Urine calcium/creatinine ratio was 0.15 . The combination of hypomagnesemia, hypokalemia, metabolic alkalosis and hypocalciuria lead to the additional diagnosis of GS.
She was given intravenous magnesium sulphate initially and later oral magnesium. Oral potassium correction was continued and indomethacin $25 \mathrm{mg}$ was given twice daily. Her magnesium and potassium levels got corrected, following which her lower limb power showed improvement (4-/5) and she was symptomatically better in terms of fatigue. She was discharged on oral magnesium supplements and indomethacin for 1 month. She was advised to drink tender coconut water daily for potassium supplementation and asked to review every 2 weeks with electrolytes report. However, she was lost for follow up.

\section{DISCUSSION}

LGMD represents a heterogeneous group of disorders involving the skeletal muscles. Both males and females are equally affected. ${ }^{1}$ They are usually autosomal recessive, but occasionally autosomal dominant or sporadic cases have been reported. ${ }^{2}$ They are classified as LGMD 1 for autosomal dominant and LGMD 2 for autosomal recessive types. There are at least 11 forms of LGMD type 2 and 6 forms of LGMD type 1. The onset is between 10 and 30 years of age. It involves the pelvic and shoulder girdle muscles and may remain confined to these areas for years. Eventually peripheral muscle wasting and weakness may occur. Contractures are unusual. Proximal reflexes are often impaired and ankle jerks are usually preserved until late in the disease. Pseudo hypertrophy may occur in calves and deltoids. Intelligence is normal. Cardiac and facial muscle involvements are extremely rare. Creatine phosphokinase levels are normal or slightly elevated. ${ }^{1}$ Treatment is mainly supportive, in the form of physiotherapy and exercise to maintain muscle strength and joint flexibility. Co-enzyme Q10 may be beneficial in these patients. ${ }^{3}$ The differential diagnosis includes polymyositis, spinal musclular atrophy and other muscular dystrophies like Duchenne, Becker etc.

GS is an autosomal recessive disorder characterized by hypokalemia, metabolic alkalosis, hypomagnesemia and hypocalciuria. ${ }^{4}$ It is caused by defective $\mathrm{NaCl}$ transport in the distal convoluted tubule. It has been linked to the gene encoding the thiazide sensitive $\mathrm{NaCl}$ cotransporter located on chromosome $16 \mathrm{q} .{ }^{5}$ These patients present at an older age. Generalized weakness is seen in severely affected patients. Cardiac arrhythmias may be present. The blood pressure is usually low or normal. Magnesium and potassium supplementation remain as the main line of treatment. Spironolactone and indomethacin may not be helpful. The long term prognosis for these patients is good. ${ }^{4}$

LGMD are rarely reported compared to other muscular dystrophies like Duchenne and Becker. However, this 
might be one of the first cases where a patient with features suggestive of LGMD had a coexisting GS, which contributed to the patient's lower limb weakness.

\section{REFERENCES}

1. Allan HR and Martin AS. Adams and Victor's Principles of Neurology. 9th ed. pp: 1372-1373.

2. Gouranga S. Limb girdle muscular dystrophy. JIACM. 2008; 9(4):302-305
3. Folkers $\mathrm{K}$ and Simonsen R. Two successful double-blind trials with coenzyme Q10 (vitamin Q10) on muscular dystrophies and neurogenic atrophies. Biochim Biophys Acta 1995; 1271: 281-286.

4. Peter $\mathrm{G}$ and Peter $\mathrm{H}$. Inherited disorders of sodium and water handling. In: Jurgen F, Richard JJ, John F, editors. Comprehensive Clinical Nephrology 4th ed. pp 577-578.

5. Simon DB, Nelson WC and Bia MJ. Gitelman's variant of Bartter's syndrome, inherited hypokalemic alkalosis, is caused by mutations in the thiazide-sensitive $\mathrm{Na}-\mathrm{Cl}$ cotransporter. Nature Genet 1996; 12: 24-30.

Authors Contribution:

RGM - Concept and design of case report, reviewed the literature, manuscript preparation, critical revision of manuscript and treating physician

Source of Support: Nil. Conflict of Interest: None. 